www.jmscr.igmpublication.org

Impact Factor (SJIF): 6.379

Index Copernicus Value: 71.58

ISSN (e)-2347-176x ISSN (p) 2455-0450

crossref DOI: _https://dx.doi.org/10.18535/jmscr/v6i3.164

Journal Of Medical Science And Clinical Research

IGM Publication

An Official Publication of IGM Publication

\title{
Study of Clinico-pathological Spectrum of Lymphadenopathy
}

\author{
Authors \\ Saini Sunita ${ }^{1}$, Mathur Kusum², Sharma Priyanka ${ }^{3}$, Pankaj JP ${ }^{4}$, Yadav Ajay \\ ${ }^{1,3}$ Resident, Department of Pathology, SMS Medical College, Jaipur, Rajasthan \\ ${ }^{2}$ Senior Professor, Department of Pathology, SMS Medical College, Jaipur, Rajasthan, India \\ ${ }^{4}$ Assistant Professor, Department of PSM, SMS Medical College, Jaipur, Rajasthan, India \\ ${ }^{5}$ Senior Professor\& Head, Department of Pathology, SMS Medical College, Jaipur, Rajasthan, India \\ Corresponding Author \\ Mathur Kusum \\ Email:drjppankaj@gmail.com
}

\begin{abstract}
Introduction: Inflammation or enlargement of lymph nodes has clinical significance because it occurs in various diseases ranges from throat infections to life threatening cancers. The lymph node involvement has an important role in cancer staging which decides treatment and prognosis. So, present study was planned to know incidence of various causes of lymphadenopathy by the histopathological examination and to find out association with age and gender.

Materials and Methods: A cross sectionalstudy was carried out in Department of Pathology, SMS Medical College, Jaipur (Rajasthan) from June 2016 to June 2017. Total 110 cases of lymphadenopathies were included and analyzed by histopathological examination of Hematoxylin and Eosin stained sections.

Results: Benign enlargement is the most common cause of lymphadenopathy. $3^{\text {rd }}$ and $4^{\text {th }}$ decade of life were commonly affected age groups with M:F ratio 1.44:1. More than half cases were of cervical lymphadenopathies. Non-neoplastic lymphadenopathy was mostly due to tuberculosis while neoplastic lymphadenopathy was due to metastatic deposits.

Conclusion: Although lymphadenopathy is mostly related with benign lesions but it necessitates a careful physical examination and follow up of patient and care should be taken in malignancy. Excisional biopsy can be used as the gold standard in the diagnosis of lymphadenopathy.

Keywords: Lymphadenopathy, Benign, Malignant, Histopathology.
\end{abstract}

\section{Introduction}

Lymph nodes are important for proper functioning of the immune system and act as filter for the foreign particles and cancer cells. Lymphadenopathy is caused by the invasion or propagation of either inflammatory cells or neoplastic cells into the node. Peripheral lymphadenopathy is a common condition in the clinical practice for physicians, paediatricians and surgeons. Easy accessibility of acquiring a sample for cytological or histological examination has made it an important component of practices of the pathologists as well. ${ }^{(1-2)}$ Patients with enlarged superficial lymph nodes sometimes require surgical biopsy for diagnosis and there have been many case series describing the pathology found at biopsy. ${ }^{(3)}$ 
Although most of the diagnoses are reactive or non-specific inflammatory conditions however a significant number of cases are composed of granulomatous inflammations, the most common cause being tuberculosis in the under privileged world while malignancies are reported as the predominant causes of lymph node enlargement in the developed countries with rarity of infections. However, an upsurge in the incidence of tuberculosis attributed to the advent and preponderance of HIV infection has been documented worldwide. ${ }^{(4-8)}$

At younger ages, individuals are more likely to have nonspecific lymphadenitis, infectious mononucleosis, rubella, and toxoplasmosis while at older ages risk of malignancy is much more. Apart from old ages the key risk factors for malignancy include firm and fixed nodal character, duration of greater than two weeks and supraclavicular location. Knowledge of these risk factors is critical in determining the management of unexplained lymphadenopathy. A metastatic solid tumor is always in the differential diagnosis of localized lymphadenopathy, particularly in older individuals. ${ }^{(9)}$

The aim and objective of the present study was to define the causes of peripheral lymphadenopathy and to know association with age and gender. It is believed that information derived from present study will form a baseline data for further research.

\section{Materials and Methods}

It was a cross sectional observational study, carried out in Department of Pathology, SMS Medical College, Jaipur (Rajasthan) from June 2016 to June 2017.Approval was taken from institutional ethics committee. Total 110 specimens of peripheral lymphadenopathies received during study period. Clinical data along with personal details of all cases were noted in a predesigned and semi-structured performa.

The tissue was fixed in $10 \%$ formalin for histopathological examination. Then paraffin embedded blocks were made in the usual manner and thin sections of 5 microns were cut by using a microtome. Sections are stained by haematoxylin $\&$ eosin stains and detailed histopathological examination was done under light microscope to diagnose and classify the lymphadenopathy. Specimens received with incomplete history or poorly fixed were excluded from the study. Data were entered in Microsoft Excel Spreadsheets 2010 and analyzed using chi square test of significance. P-value $<0.05$ was considered as the level of significance.

\section{Results}

In the present study, out of 110 specimens 65 $(59.09 \%)$ were male and $45(40.91 \%)$ were female. The maximum cases $49(44.55 \%)$ were in 21-40 years age group, followed by $24(21.82 \%)$ in 41-60 years, $22(20.00 \%)$ in $0-20$ years, 15 $(13.64 \%)$ in $>60$ years. While analysing according to site of lymphadenopathy, it was found that highest $58(52.73 \%)$ cases were presented with cervical lymphadenopathy, followed by 12 $(10.91 \%)$ cases axillary lymphadenopathy, 10 $(9.09 \%)$ cases inguinal and mesenteric lymphadenopathy each,8 (7.27\%) cases post auricular lymphadenopathy and $5(4.55 \%)$ cases supraclavicular lymphadenopathy. 7 (6.36\%) cases were presented with generalized lymphadenopathy (Figure-1).

Table-1 shows the gender distribution between noneoplastic and neoplastic lymphadenopathy where both the groups showed the male predominance $(53.33 \%$ in Non-neoplastic and $71.43 \%$ in neoplastic lymphadenopathy).

Majority of cases 75 (68.18\%) were nonneoplastic while $35(31.82 \%)$ cases were neoplastic lymphadenopathy. Among nonneoplastic lymphadenopathy $40(53.33 \%)$ cases were tuberculosis, reactive hyperplasia 20 (26.67\%), chronic non-specific lymphadenitis 4 $(5.33 \%)$, sinus histiocytosis $3(4.00 \%)$, necrotizing granulomatous lymphadenopathy $2(2.67 \%)$, acute suppurative lymphadenitis 1(1.33\%), KikuchiFuzimoto disease 2(2.67\%), Castleman disease $2(2.67 \%)$. Female predominance was observed in 
tuberculosis, reactive hyperplasia, necrotizing granulomatous lymphadenitis and Rosai-Dorfman disease while male predominance was observed in chronic non- specific lymphadenopathy, sinus histiocytosis, Kikuchi disease, Castleman disease and acute suppurative lymph noditis (Table-2).

It was observed that highest affected age group was 21-40 years in tubercular lymphadenopathy and reactive lymphadenopathy while in chronic non-specific lymphadenitis it was 0-40 years, in sinus histiocytosis41-60 years, inacute suppurative lymphadenitis and Rosai-Dorfman disease 41-60 years, Kikuchi and Castleman disease 21-40 years and in necrotizing granulomatous diseases it was> 41 years (Table-3).

In neoplastic lymphadenopathy lymphomas were $15(42.86 \%)$ and metastatic carcinomas were 20 (57.14\%). Out of 15 cases of lymphomas 9 were Hodgkin lymphoma and 6 were non-Hodkin lymphoma whileout of 20 cases of metastatic carcinoma, maximum number of cases were of squamous cell carcinoma (9) followed by adenocarcinoma (8) and 1 each of mucoepidermoid carcinoma, small cell carcinoma and malignant melanoma. Male predominance was observed in Hodgkin lymphoma, nonHodgkin lymphoma, metastatic squamous cell carcinoma and mucoepidermoid carcinoma. Female predominance was observed in metastatic adenocarcinoma and metastatic melanoma (Table4).

The present study showed that highest cases of Hodgkin lymphomas were in 21-40 years age group and lowest were in 0-20 years. Maximum number of non-Hodgkin lymphomas were in 0-20 years age group while highest number of metastatic carcinoma were in 41-60 years. Metastatic carcinomas were not found in age group 0-20 years (Table-5).

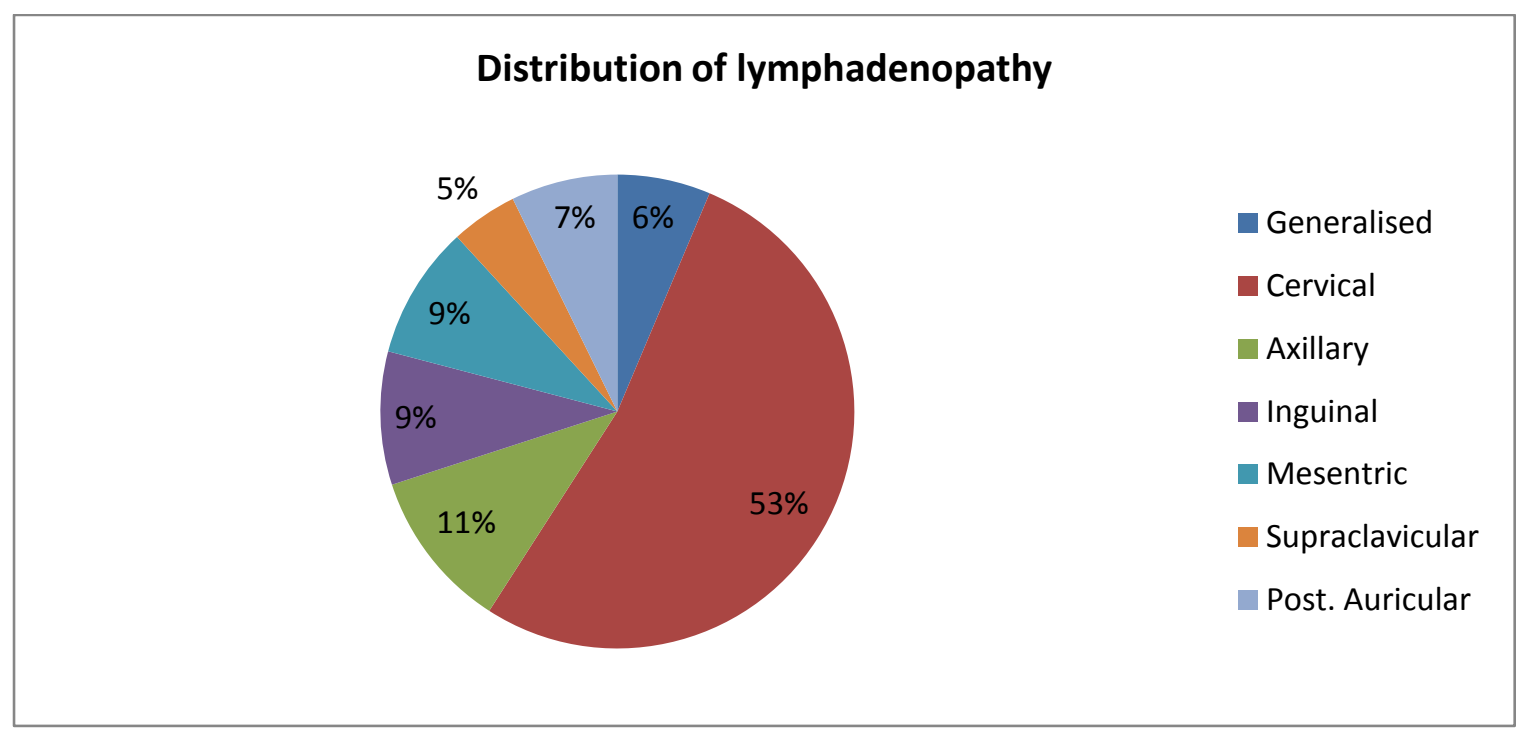

Figure: 1 Distribution of lymphadenopathy according to site.

Table-1: Gender wise distribution of lymphadenopathy $(n=110)$

\begin{tabular}{|l|c|c|}
\hline Diagnosis & Male & Female \\
\hline Non-neoplastic $(\mathrm{n}=75)$ & $40(53.33)$ & $35(46.67)$ \\
\hline Neoplastic $(\mathrm{n}=35)$ & $25(71.43)$ & $10(28.57)$ \\
\hline
\end{tabular}


Table-2: Gender wise distribution of different non-neoplastic lymphadenopathy $(n=75)$

\begin{tabular}{|c|c|c|c|}
\hline Histopathological Diagnosis & $\begin{array}{c}\text { Male } \\
\text { n (\%) }\end{array}$ & $\begin{array}{c}\text { Female } \\
\text { n }(\%)\end{array}$ & $\begin{array}{l}\text { Total } \\
\text { n }(\%)\end{array}$ \\
\hline Tuberculosis & $\begin{array}{c}19 \\
(25.33)\end{array}$ & $\begin{array}{c}21 \\
(28.00)\end{array}$ & $\begin{array}{c}40 \\
(53.33)\end{array}$ \\
\hline Reactive Hyperplasia & $\begin{array}{c}9 \\
(12.00) \\
\end{array}$ & $\begin{array}{c}11 \\
(14.67)\end{array}$ & $\begin{array}{c}20 \\
(26.67)\end{array}$ \\
\hline Chronic Non-specific Lymphadenitis & $\begin{array}{c}4 \\
(5.33) \\
\end{array}$ & $\begin{array}{c}0 \\
(0.00) \\
\end{array}$ & $\begin{array}{c}4 \\
(5.33) \\
\end{array}$ \\
\hline Sinus Histiocytosis & $\begin{array}{c}3 \\
4.00)\end{array}$ & $\begin{array}{c}0 \\
(0.00)\end{array}$ & $\begin{array}{c}3 \\
(4.00)\end{array}$ \\
\hline Necrotizing Gronulomatous Lymphoma & $\begin{array}{c}0 \\
(0.00) \\
\end{array}$ & $\begin{array}{c}2 \\
(2.67) \\
\end{array}$ & $\begin{array}{c}2 \\
(2.67) \\
\end{array}$ \\
\hline Kikuchi Fuzimoto Disease & $\begin{array}{c}2 \\
(2.67)\end{array}$ & $\begin{array}{c}0 \\
(0.00) \\
\end{array}$ & $\begin{array}{c}2 \\
(2.67) \\
\end{array}$ \\
\hline RosaiDorfman Disease & $\begin{array}{c}0 \\
(0.00)\end{array}$ & $\begin{array}{c}1 \\
(1.33)\end{array}$ & $\begin{array}{c}1 \\
(1.33)\end{array}$ \\
\hline Castleman Disease & $\begin{array}{c}2 \\
(2.67)\end{array}$ & $\begin{array}{c}0 \\
(0.00)\end{array}$ & $\begin{array}{c}2 \\
(2.67)\end{array}$ \\
\hline Acute Suppurative Lymphadenitis & $\begin{array}{c}1 \\
(1.33)\end{array}$ & $\begin{array}{c}0 \\
(0.00)\end{array}$ & $\begin{array}{c}1 \\
(1.33)\end{array}$ \\
\hline Total & $\begin{array}{c}40 \\
(53.33)\end{array}$ & $\begin{array}{c}35 \\
(46.67)\end{array}$ & $\begin{array}{c}75 \\
(100)\end{array}$ \\
\hline
\end{tabular}

Table-3: Age wise distribution of different non-neoplastic lymphadenopathy $(n=75)$

\begin{tabular}{|c|c|c|c|c|c|}
\hline \multirow[b]{2}{*}{ Histopathological Diagnosis } & \multicolumn{5}{|c|}{ Age groups (in Years) } \\
\hline & $\begin{array}{c}0-20 \\
\text { n }(\%)\end{array}$ & $\begin{array}{l}21-40 \\
\text { n }(\%)\end{array}$ & $\begin{array}{l}41-60 \\
\text { n }(\%)\end{array}$ & $\begin{array}{c}>60 \\
\mathrm{n}(\%)\end{array}$ & $\begin{array}{l}\text { Total } \\
\text { n }(\%)\end{array}$ \\
\hline Tuberculosis & $\begin{array}{c}8 \\
(10.67)\end{array}$ & $\begin{array}{c}23 \\
(30.67)\end{array}$ & $\begin{array}{c}7 \\
(9.33)\end{array}$ & $\begin{array}{c}2 \\
(2.67) \\
\end{array}$ & $\begin{array}{c}40 \\
(53.33)\end{array}$ \\
\hline Reactive Hyperplasia & $\begin{array}{c}7 \\
(9.33) \\
\end{array}$ & $\begin{array}{c}8 \\
(10.67) \\
\end{array}$ & $\begin{array}{c}3 \\
(4.00) \\
\end{array}$ & $\begin{array}{c}2 \\
(2.67)\end{array}$ & $\begin{array}{c}20 \\
(26.67)\end{array}$ \\
\hline Chronic Non-specific Lymphadenitis & $\begin{array}{c}2 \\
(2.67) \\
\end{array}$ & $\begin{array}{c}2 \\
(2.67) \\
\end{array}$ & $\begin{array}{c}0 \\
(0.00) \\
\end{array}$ & $\begin{array}{c}0 \\
(0.00) \\
\end{array}$ & $\begin{array}{c}4 \\
(5.33) \\
\end{array}$ \\
\hline Sinus Histiocytosis & $\begin{array}{c}0 \\
(0.00)\end{array}$ & $\begin{array}{c}0 \\
(0.00)\end{array}$ & $\begin{array}{c}3 \\
(4.00)\end{array}$ & $\begin{array}{c}0 \\
(0.00) \\
\end{array}$ & $\begin{array}{c}3 \\
(4.00)\end{array}$ \\
\hline Necrotizing Gronulomatous Lymphoma & $\begin{array}{c}0 \\
(0.00)\end{array}$ & $\begin{array}{c}0 \\
(0.00)\end{array}$ & $\begin{array}{c}1 \\
(1.33)\end{array}$ & $\begin{array}{c}1 \\
(1.33)\end{array}$ & $\begin{array}{c}2 \\
(2.67)\end{array}$ \\
\hline Kikuchi Fuzimoto Disease & $\begin{array}{c}0 \\
(0.00)\end{array}$ & $\begin{array}{c}2 \\
(2.67)\end{array}$ & $\begin{array}{c}0 \\
(0.00) \\
\end{array}$ & $\begin{array}{c}0 \\
(0.00) \\
\end{array}$ & $\begin{array}{c}2 \\
(2.67) \\
\end{array}$ \\
\hline RosaiDorfman Disease & $\begin{array}{c}0 \\
(0.00)\end{array}$ & $\begin{array}{c}0 \\
(0.00)\end{array}$ & $\begin{array}{c}1 \\
(1.33)\end{array}$ & $\begin{array}{c}0 \\
(0.00)\end{array}$ & $\begin{array}{c}1 \\
(1.33)\end{array}$ \\
\hline Castleman Disease & $\begin{array}{c}0 \\
(0.00)\end{array}$ & $\begin{array}{c}2 \\
(2.67) \\
\end{array}$ & $\begin{array}{c}0 \\
(0.00)\end{array}$ & $\begin{array}{c}0 \\
(0.00) \\
\end{array}$ & $\begin{array}{c}2 \\
(2.67)\end{array}$ \\
\hline Acute Suppurative Lymphadenitis & $\begin{array}{c}0 \\
(0.00)\end{array}$ & $\begin{array}{c}0 \\
(0.00)\end{array}$ & $\begin{array}{c}1 \\
(1.33)\end{array}$ & $\begin{array}{c}0 \\
(0.00)\end{array}$ & $\begin{array}{c}1 \\
(1.33)\end{array}$ \\
\hline Total & $\begin{array}{c}17 \\
(22.67)\end{array}$ & $\begin{array}{c}37 \\
(49.33)\end{array}$ & $\begin{array}{c}16 \\
(21.33)\end{array}$ & $\begin{array}{c}5 \\
(6.67)\end{array}$ & $\begin{array}{c}75 \\
(100)\end{array}$ \\
\hline
\end{tabular}


Table-4: Gender wise distribution of different neoplastic lymphadenopathy $(n=35)$

\begin{tabular}{|l|c|c|c|}
\hline Histopathological Diagnosis & $\begin{array}{c}\text { Male } \\
\mathbf{n}(\%)\end{array}$ & $\begin{array}{c}\text { Female } \\
\mathbf{n}(\boldsymbol{\%})\end{array}$ & $\begin{array}{c}\text { Total } \\
\mathbf{n}(\boldsymbol{\%})\end{array}$ \\
\hline Hodgkin Lymphoma & 8 & 1 & 9 \\
& $(22.86)$ & $(2.86)$ & $(25.71)$ \\
\hline Non-Hodgkin Lymphoma & 6 & 0 & 6 \\
& $(17.14)$ & $(0.00)$ & $(17.14)$ \\
\hline Metastatic Squamous Cell Carcinoma & 6 & 3 & 9 \\
$(17.14)$ & $(8.57)$ & $(25.71)$ \\
\hline Metastatic Adenocarcinoma & 2 & 6 & 8 \\
\multirow{2}{*}{ Metastatic Muco-epidermoid Carcinoma } & $(5.71)$ & $(17.14)$ & $(22.86)$ \\
\hline \multirow{2}{*}{ Metastatic Small Cell Carcinoma } & $(2.86)$ & $(0.00)$ & $(2.86)$ \\
\hline \multirow{2}{*}{ Metastatic Melanoma } & 1 & 0 & 1 \\
& $(2.86)$ & $(0.00)$ & $(2.86)$ \\
\hline \multirow{2}{*}{ Total } & 0 & 1 & 1 \\
& $(0.00)$ & $(2.86)$ & $(2.86)$ \\
\hline
\end{tabular}

Table-5: Age wise distribution of different neoplastic lymphadenopathy $(\mathrm{n}=35)$

\begin{tabular}{|c|c|c|c|c|c|}
\hline \multirow[b]{2}{*}{ Histopathological Diagnosis } & \multicolumn{5}{|c|}{ Age groups (in Years) } \\
\hline & $\begin{array}{c}0-20 \\
\text { n }(\%)\end{array}$ & $\begin{array}{l}21-40 \\
\text { n }(\%)\end{array}$ & $\begin{array}{l}41-60 \\
\text { n }(\%)\end{array}$ & $\begin{array}{c}>60 \\
n(\%)\end{array}$ & $\begin{array}{l}\text { Total } \\
\text { n }(\%)\end{array}$ \\
\hline Hodgkin Lymphoma & $\begin{array}{c}1 \\
(2.86)\end{array}$ & $\begin{array}{c}6 \\
(17.14)\end{array}$ & $\begin{array}{c}0 \\
(0.00)\end{array}$ & $\begin{array}{c}2 \\
(5.71)\end{array}$ & $\begin{array}{c}9 \\
(25.71)\end{array}$ \\
\hline Non-Hodgkin Lymphoma & $\begin{array}{c}3 \\
(8.57)\end{array}$ & $\begin{array}{c}0 \\
(0.00)\end{array}$ & $\begin{array}{c}1 \\
(2.86)\end{array}$ & $\begin{array}{c}2 \\
(5.71)\end{array}$ & $\begin{array}{c}6 \\
(17.14)\end{array}$ \\
\hline Metastatic Squamous Cell Carcinoma & $\begin{array}{c}0 \\
(0.00)\end{array}$ & $\begin{array}{c}0 \\
(0.00)\end{array}$ & $\begin{array}{c}4 \\
(11.43)\end{array}$ & $\begin{array}{c}5 \\
(14.29)\end{array}$ & $\begin{array}{c}9 \\
(25.71)\end{array}$ \\
\hline Metastatic Adenocarcinoma & $\begin{array}{c}0 \\
(0.00)\end{array}$ & $\begin{array}{c}1 \\
(2.86)\end{array}$ & $\begin{array}{c}6 \\
(17.14)\end{array}$ & $\begin{array}{c}1 \\
(2.86)\end{array}$ & $\begin{array}{c}8 \\
(22.86)\end{array}$ \\
\hline Metastatic Muco-epidermoid Carcinoma & $\begin{array}{c}0 \\
(0.00)\end{array}$ & $\begin{array}{c}1 \\
(2.86)\end{array}$ & $\begin{array}{c}0 \\
(0.00)\end{array}$ & $\begin{array}{c}0 \\
(0.00) \\
\end{array}$ & $\begin{array}{c}1 \\
(2.86)\end{array}$ \\
\hline Metastatic Small Cell Carcinoma & $\begin{array}{c}0 \\
(0.00)\end{array}$ & $\begin{array}{c}0 \\
(0.00)\end{array}$ & $\begin{array}{c}0 \\
(0.00)\end{array}$ & $\begin{array}{c}1 \\
(2.86)\end{array}$ & $\begin{array}{c}1 \\
(2.86)\end{array}$ \\
\hline Metastatic Melanoma & $\begin{array}{c}0 \\
(0.00)\end{array}$ & $\begin{array}{c}0 \\
(0.00)\end{array}$ & $\begin{array}{c}1 \\
(2.86)\end{array}$ & $\begin{array}{c}0 \\
(0.00)\end{array}$ & $\begin{array}{c}1 \\
(2.86)\end{array}$ \\
\hline Total & $\begin{array}{c}4 \\
(11.43)\end{array}$ & $\begin{array}{c}8 \\
(22.86)\end{array}$ & $\begin{array}{c}12 \\
(34.29)\end{array}$ & $\begin{array}{c}11 \\
(31.43)\end{array}$ & $\begin{array}{c}35 \\
(100)\end{array}$ \\
\hline
\end{tabular}

\section{Discussion}

Lymphadenopathy is a very common clinical manifestation of many disease including infection, primary and secondary malignant lesions. The present study comprised of 110 cases of lymph node biopsy received in department of Pathology, SMS Medical College Jaipur during January 2016 to September 2017.

In the present study, 65 (59.09\%) were male and $45(40.91 \%)$ were female with male to female ratio 1.4:1 revealing male preponderance. The male to female ratio was $1.2: 1$ in study conducted by Adesuwa $\mathrm{N}$ et al. $2006^{(10)}, 1.14: 1$ by Abdulkadar Mohammed Albasari et al. (2014) ${ }^{(2)}$ and 1.23:1 by
Inayatullah khan et al. $(2015)^{(11)}$. So preponderance of male over female in lymphadenopathy in current study was almost in accordance with previous studies.

In present study maximum lymph node involved were cervical $(52.73 \%)$ followed by axillary $(10.91 \%)$, inguinal and mesenteric node $(9.09 \%$ each) which was concordance with the study of Adesuwa $\mathrm{N}$ et al., $2006^{(10)}$ (58.5\% cervical lymph nodes), S Chhabra et al., $2005^{(12)}$ (cervical $69.2 \%$, axillary 19.6\%), Abdulkadar Mohammed Albasari et al., 2014 ${ }^{(2)}$ (cervical $30.4 \%$, axillary $9.7 \%$ ) and Nader Murad at el., 2012(13) (cervical 70\%, axillary 67\%). Most common involvement of 
cervical lymph node is due to infection of tonsil, adenoids, Waldayer's ring and portal of entry may be inhalation of M. tuberculosis.

In present study $68.18 \%$ cases were nonneoplastic and $31.82 \%$ were neoplastic lymphadenopathy which were concordance with the previous study by $\mathrm{S}$ Chhabra et al., $2005^{(12)}$ (non-neoplastic lesion $67.6 \%$ and neoplastic $32.4 \%$ ) and Nadiramajid at el. $2013^{(3)}$ (nonneoplastic lesion $55.7 \%$ and neoplastic lesion $44.3 \%$ ). In the present study, out of 75 nonneoplastic lesion, $40(53.3 \%)$ were male and 35 $(46.6 \%)$ were female while out of 35 neoplastic lesion $25(71.5 \%)$ were male $10(28.5 \%)$ were female with male predominance in both neoplastic and non-neoplastic lesion. Similar results were observed by Nadiramajid at el. $2013^{(3)}$ and Esra Akyuzozkan at el. 2015 $5^{(14)}$.

In non-neoplastic lymphadenopathy tubercular lymphadenitis was most common cause (36.3\%). The study conducted by Adesuwa $\mathrm{N}$ et al., $2006^{(10)}$ also showed that tubercular lymphadenitis was major causes of lymph node enlargement constituting $26.7 \%$. Inayatullah khan et al. $(2015)^{(11)}$ also found the same result, tubercular lymphadenopathy $36.2 \%$.Increase prevalence of tubercular lymphadenitis was due to poverty, overcrowding and poor sanitary conditions. Maximum number of cases of tubercular lymphadenitis (23 out of 40) were found in 21-40 years of age group which was similar to previous study by Abdulkadar Mohammed Albasari et al., $2014^{(2)}$, where he found 26 tubercular lymphadenopathy out of 41 cases in age group 2039 years.

In present study, $26.67 \%$ cases of reactive lymphadenopathy were found. This was very close to previous study by Adesuwa $\mathrm{N}$ et al., $2006^{(10)}$ (19\% reactive lymphadenopathy) and Inayatullah khan et al. $(2015)^{(11)}(19.3 \%$ of reactive lymphadenitis). Maximum number of cases of reactive lymphadenopathy (8 out of 20) were found in age group 21-40 years which was similar to study by Abdulkadar Mohammed Albasari et al., 2014 ${ }^{(2)}$.
In our study two cases of Kikuchi-Fuzimoto disease, two cases Castleman disease and one case Rosai-Dorfman disease were found. Kikuchi disease also known as Histiocytic necrotizing lymphadenitis (HNL); is an autoimmune, selflimiting, benign disease. Histologically lymph nodes showed partial architectural effacement by large discrete areas of necrosis with abundant nuclear debris surrounded by lymphocytes, histiocytes and plasmacytoid monocytes. ${ }^{(15)}$ Castleman disease also called angiofollicular lymphoid hyperplasia. Histologically these cases show partial effacement of lymph node architecture by large follicles having atrophic germinal centres. The Centre of follicles and interfollicular areas showed increased vascularity with hyalinization of vascular walls, at places vessels were seen entering the germinal centers from interfollicular zone. ${ }^{(16)}$ Rosai-Dorfman disease, also called sinus histiocytosis with massive lymphadenopathy (SHML). Histologically shows dilated sinuses, large histiocytes with intact phagocytosed lymphocytes (emperipolesis), heavily infiltrated with large histiocytes, lymphocytes and plasma-cells in the background. (17)

In present study, out of $35(31.82 \%)$ cases of neoplastic lymphadenopathy $20(18.18 \%)$ cases were metastatic carcinoma while in previous studies by Adesuwa $\mathrm{N}$ et al., 2006 ${ }^{(10)}$ and Inayatullah khan et al. (2015) $)^{(11)}$ they had found $26.5 \%$ and $29.3 \%$ cases of neoplastic lymphadenopathy respectively. Metastatic carcinoma was mostly in $>40$ years of age with slight female predominance (10 cases female and 9 case male). Shrestha AK et al., 2009 ${ }^{(18)}$, U.Engzell et al. ${ }^{(19)}$ and William L Betsill et al. ${ }^{(20)}$ also found in their studies that $80-90 \%$ cases of metastatic lymph node involvement was among 41-80 years.

In present study, lymphoma patients showed wide age distribution 9-75 years. Total 15 (13.63\%) cases of lymphoma were found with male predominance. This was slight lower than $19.1 \%$ reported by Pindiga et al., $1999^{(21)}$. Most of cases 
of Hodgkin lymphoma were 21-40 years age group and all cases of Hodgkin lymphoma were classical type. Classic Hodgkin lymphoma (CHL) is a monoclonal lymphoid neoplasm composed of mononuclear Hodgkin and multinucleated Hodgkin Reed-Sternberg (HRS) cells residing in an infiltrate containing a variable mixture of nonneoplastic small lymphocytes, eosinophils, neutrophils, histiocytes, plasma cells, fibroblasts and collagen fibers.

In non-Hodgkin lymphoma (7 cases), 5 cases were B-cell type and 2 cases were T-cell type. In B-cell type lymphoma 2 case NHL-CLL/SLL, 2 cases NHL-B cell type, high grade and one case of Burkit lymphoma were found with wide range of age group. Non Hodgkin lymphoma is clonal lymphoproliferative disorder. It is heterogenous neoplasms and $80 \%$ are B cell, NK are rare.

\section{Conclusion}

Lymphadenopathy is a common problem and necessitates a careful physical examination and follow up. Laboratory and imaging methods should be used in the differential diagnosis when necessary. Excisional biopsy is used as gold standard in the diagnosis of lymphadenopathy. Although lymphadenopathy is mostly related with benign lesions, care should be taken in malignancy.

\section{References}

1. Elmore SA. Enhanced histopathology of lymph nodes. Toxicolpathol. 2006;34(5): 634-47.

2. Abdulkader Mohammed Albasri, Abeer Abdalla E.Pattern of Lymph Node Pathology in Western Saudi Arabia. Asian Pac J Cancer.2014;15(11):4677-4681.

3. NadiraMajid, MA SamadTalukder et al. Histodemography of lymphadenopathy. Delta med col J. Jan 2013;1(1):8-12.

4. Fazal-I-wahid, Habib-Ur-Rehman, Ahmad I. Extrapulmonary tuberculosis in patients with cervical lymphadenopathy. J Pak Med Assoc. 2013;63:1094-7.
5. Freidig EE, McClure SP, Wilson WR, Banks PM, Washington JA. Clinical histologic-microbiologic analysis of 419 lymph node biopsy specimens. Rev Infect Dis. 1986;8:322-8.

6. Sriwatanawongsa V, Cardoso R, Chang P Incidence of malignancy in peripheral lymph node biopsy. Am Surg. 1985; 51:587-90.

7. Markowitz N, Hansen NI, Hopewell PC, Glassroth J, Kvale PA, Mangura BT, et al. Incidence of tuberculosis in the United States among HIV infected persons. Ann Intern Med. 1997;126:123-32.

8. Bem C, Patil PS, Bharucha H, Namaambo $\mathrm{K}$, Luo N. Importance of human immunodeficiency virus-associated lymphadenopathy and tuberculous lymphadenitis in patients undergoing lymph node biopsy in Zambia. Br J Surg. 1996;83:75-8.

9. Pieter J Tenis, Omgo E Niewag et al. History of senital node and validation of the technique .breast cancer res. 2001;3(2):109-112.

10. Adesuwa N, Olu-Eddo et al. Peripheral lymphadenopathy in Nigerian adults. J Pak Med Assoc. 2006;56(9):405-8.

11. Inayatullah Khan, Ehsan Gul Khattak. Cervical Lymphadenopathy: An Audit Of 116 Cases. Isra Medical Journal. Jan - Mar 2015;7(1):6-9.

12. S Chhabra, H Mohan, A Bal. A Retrospective Histological Evaluation of Non-neoplastic Superficial Lymphadenopathy. The Internet Journal of Internal Medicine. 2005;6(1):1-5.

13. Nader Morad, FCAP, TarekMalatani, FRCS et al. Peripheral lymphadenopathy as a primary presenting sign: A study of 324 cases from Asia region. Annals of Saudi medicine. 2012;12:72-5.

14. Esra Akyüz Özkan, Ceren Canbey Göret. Evaluation of peripheral lymphadenopathy with excisional biopsy: six-year 
experience. Int J ClinExpPathol. 2015;8(11):15234-15239.

15. Nazer MA, Salem AH et al. Excision biopsy in peripheral lymphadenopathy in children in a community hospital. Annals of Saudi Medicine. 2003;23:410-13.

16. Hirachand S, Lakhey M et al. Evaluation of fine needle aspiration cytology of lymph nodes in Kathmandu Medical College, Teaching hospital. Kathmandu Univ Med J (KUMJ). 2009 Apr-Jun;7(26):139-42.

17. Ojo BA, Buhari MO et al. Surgical lymph node bopsies in Llorin teaching Hospital, Llorin, Nigeria. Niger Postgrad Med J. 2005;12(4):299-304.

18. Shrestha AK, Chalise PR et al. Lymph node biopsies; Hospital based retrospective study. J Nepal med assoc. 2009;48(176);306-9.

19. Engzell U, jaksobsson P.A. Aspiration biopsy of metastatic carcinoma in lymph node of the neck. actaOtolaryng. 2009;72:138-147.

20. Betsill William L and Hajdu Steven I. Percutaneous aspiration biopsy of lymph nodes. A.J.C.P. 1980;23(4):471-479.

21. Pindiga UH, Dogo D. Histopathology of primary peripheral lymphadenopathy in North Eastern Nigeria. Nig J Surg Res. 1999; 1:68 -71. 